Original Research

\title{
Single-Center Outcomes of Heart Transplantation in Patients with Pulmonary Hypertension Bridged with Mechanical Circulatory Support
}

Dominic Emerson ${ }^{1,2}$, Jon Kobashigawa ${ }^{1}$, Ryan Levine ${ }^{1}$, Jamie Moriguchi ${ }^{1}$, Dominick Megna ${ }^{1,2}$, Joshua Chung ${ }^{1,2}$, Danny Ramzy ${ }^{1,2}$, Alfredo Trento ${ }^{1,2}$, Fardad Esmailian ${ }^{1,2, *}$

1. Cedars-Sinai Heart Institute, Los Angeles, CA, USA; E-Mails: dominic.emerson@cshs.org; jon.kobashigawa@cshs.org; $\quad$ ryan.levine@csmns.org; moriguchiJ@csmns.org;

Dominick.Megna@cshs.org; JOSChung@llu.edu; Danny.ramzy@cshs.org; Alfredo.trento@cshs.org; Fardad.esmailian@cshs.org

2. Department of Cardiac Surgery, Cedars-Sinai Medical Center, Los Angeles, CA, USA

* Correspondence: Fardad Esmailian; Email: Fardad.esmailian@cshs.org

Academic Editor: Yasuhiko Sugawara

Special Issue: Lung and Heart Transplantation

OBM Transplantation

2020, volume 4, issue 3

doi:10.21926/obm.transplant.2003114
Received: April 14, 2020

Accepted: July 01, 2020

Published: July 06, 2020

\begin{abstract}
In advanced heart failure, orthotopic heart transplantation (OHT) remains the standard for definitive treatment. Patients with heart failure and concomitant pulmonary hypertension (PH) represent a challenging subgroup, with poor outcomes following OHT. Recently, Mechanical Circulatory Support (MCS) devices have been utilized as a bridge to OHT for these patients. Patients with MCS devices who underwent OHT were evaluated at a single center. Patients were stratified into three groups, depending on baseline pulmonary vascular resistance (PVR). Groups were analyzed for outcomes. One hundred twenty three patients were identified. Of these, 39 had a PVR $\leq 1.6,50$ had a PVR $>1.6$ and $\leq 3$, and 34 had a PVR $>3$. Demographics were generally
\end{abstract}

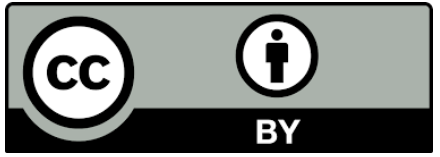

(C) 2020 by the author. This is an open access article distributed under the conditions of the Creative Commons by Attribution License, which permits unrestricted use, distribution, and reproduction in any medium or format, provided the original work is correctly cited. 
similar. Tricuspid valve regurgitation (TR) was significantly higher among patients with worse baseline PVR $(p=0.016)$. Survival was similar at both one and three years. On subsequent analysis, moderate/severe TR was associated with worse survival. MCS implantation as a bridge to OHT has good one and three-year outcomes among patients with increasing pre-MCS PVR. However, patients with severe PH prior to MCS have an increased rate of TR. Among these patients, moderate-severe TR after OHT has an associated risk of early death.

\section{Keywords}

Orthotopic heart transplantation; pulmonary hypertension; left ventricular assist device

\section{Introduction}

For patients with advanced heart failure, orthotopic heart transplantation (OHT) remains the gold standard for definitive treatment. While OHT outcomes have generally improved and the potential recipient pool broadened, patients who present with heart failure in the setting of concomitant pulmonary hypertension $(\mathrm{PH})$ continue to represent a challenging subgroup. Poor outcomes in patients with PH following OHT have been noted since the early days of heart transplantation, with the resultant strain on the non-conditioned right ventricle (RV) often leading to RV failure and subsequent graft failure $[1,2]$ These observed outcomes have led to the establishment of PH as a relative contraindication to $\mathrm{OHT}[1]$, though the cutoff level for limiting candidacy is variable between centers.

In an effort to address these challenges, mechanical circulatory support (MCS), including left ventricular assist device (LVAD), biventricular assist device (BiVAD), or total artificial heart (TAH) implantation has been utilized to fully unload the left ventricle (LV) and allow pulmonary pressures to improve as a bridge to transplantation. This approach has been noted to improve candidacy in this population [3-5]. To better establish outcomes for this challenging population, we evaluated the impact of pre-OHT pulmonary vascular resistance (PVR) in patients who underwent $\mathrm{OHT}$ following MCS placement at a single large-volume center.

\section{Materials and Methods}

This study was reviewed and approved by the Cedars-Sinai Medical Center Institutional review board.

\subsection{Patient Population}

All heart transplant recipients from 2010 to 2017 who were bridged to transplant with MCS were identified from a prospectively maintained institutional database. Patients were stratified based on PVR into three groups: normal PVR (Group A: PVR $\leq 1.6$ wood units), moderately elevated PVR (Group 
B: PVR between 1.6 and 3 wood units), and severely elevated PVR (Group C: PVR > 3 wood units). Data were then retrospectively analyzed.

\subsection{Evaluation of Pulmonary Hypertension}

All patients in this study underwent right heart catheterization (RHC) during initial workup prior to placement of MCS, as is the standard for our patients. During this procedure (and at any other subsequent RHC prior to OHT) central venous pressure (CVP), pulmonary arterial pressure (PAP), mean PAP, PVR, and cardiac output (CO)/ cardiac index $(\mathrm{Cl})$ were measured.

\subsection{Mechanical Assist Devices}

Patients in this study received one of the following MCS devices- a LVAD (Heart Mate II (Abbott, Chicago, IL, USA), Heart Mate III (Abbott, Chicago, IL, USA), or Heart Ware HVAD (Medtronic, MN, USA)), BiVAD using right and left Thoratec PVAD (Thoratec, Pleasanton, CA), or a TAH (Syncardia, Tucson, AZ). All devices were implanted using the standard technique, and in accordance with manufacturer guidelines.

\subsection{Endpoints}

Post-transplant endpoints included 1-year survival, 1-year freedom from the development of moderate-severe tricuspid regurgitation (TR), 1-year freedom from cardiac allograft vasculopathy (CAV) as defined by stenosis $\geq 30 \%$ by angiography, 1-year freedom from non-fatal major adverse cardiac events (NF-MACE: myocardial infarction, new congestive heart failure, percutaneous coronary intervention, implantable cardioverter defibrillator/pacemaker implant, stroke), 1-year creatinine, 1year total bilirubin, and 1-year freedom from any-treated rejection (ATR), acute cellular rejection $(A C R)$, and antibody-mediated rejection (AMR).

\subsection{Statistical Analysis}

Continuous variables were reported as mean \pm standard deviation, and categorical variables as a percentage. Comparison between groups was performed using the ANOVA test for continuous variables and the Pearson's Chi-Squared tests for categorical variables as a percentage, as appropriate. Survival and freedom from TR analysis were performed using the Kaplan-Meier method and compared between groups using log-rank tests. A p-values less than 0.05 was considered significant throughout. All statistical analyses were performed using SPSS software, Version 22 (IBM Corp., Armonk, NY).

\section{Results}

In total 123 patients were identified that fit the established criteria during this time period. Of these, 39 had a PVR $\leq 1.6$ (group A), 50 had a PVR >1.6 and $\leq 3$ (group B), and 34 had a PVR >3 (group C). Baseline demographics for these patients are listed in Table 1, and were generally similar between 
groups, with the exception of female sex which was lower in group $B(25.6 \%, 6 \%$, and $32.4 \%$ for groups $A, B$, and $C$, respectively, $p=0.006$ ). Transplant factors between groups (Table 2) were also generally similar, except pre-MCS MPAP, which was, as anticipated, greater as PVR increased (28.3, 36.1 , and 41.8 for groups $A, B$, and C, respectively, $p<0.001$ ).

Endpoints evaluated are shown in Table 3 and were statistically significant for differences in oneyear freedom from moderate-severe TR $(94.9 \%, 82.0 \%$, and $70.6 \%$, for groups $A, B$, and $C$, respectively, $p=0.016$; graphically seen in Figure 1 ), and one-year freedom from any-treated rejection $(92.3 \%, 84.0 \%$, and $100 \%$, for groups A, B, and C, respectively, $p=0.016)$. Survival at one-year was not statistically different between groups (Table 3 and Figure 2). An extended analysis of survival was undertaken at the three-year mark, and this also continued to be statistically similar between groups (Figure 3).

Additional survival subgroup analyses evaluated included one-year survival as stratified by TR (Table 4), and one-years survival in patients with moderate-severe TR as stratified by group (Table 5). Survival at one year stratified by TR (Table 4) was significantly worse in those with moderate-severe TR (96.1\% vs $76.2 \%, p<0.001)$.

Table 1 Patient demographics

\begin{tabular}{lllll}
\hline Demographics & $\begin{array}{l}\text { Group A } \\
\text { PVR } \leq 1.6 \\
(\mathbf{n}=\mathbf{3 9})\end{array}$ & $\begin{array}{l}\text { Group B } \\
\mathbf{1 . 6}<\text { PVR } \leq \mathbf{3} \\
(\mathbf{n}=\mathbf{5 0})\end{array}$ & $\begin{array}{l}\text { Group C } \\
\text { PVR }>\mathbf{3} \\
(\mathbf{n}=\mathbf{3 4})\end{array}$ & p value \\
\hline Recipient Age, years & $53.8 \pm 11.0$ & $51.5 \pm 13.0$ & $52.3 \pm 11.8$ & 0.683 \\
Recipient Sex, \% Female & $25.6 \%$ & $6.0 \%$ & $32.4 \%$ & 0.006 \\
Recipient BMI (kg/m $\left.{ }^{2}\right)$ & $25.8 \pm 5.3$ & $27.8 \pm 4.4$ & $26.2 \pm 4.9$ & 0.105 \\
Race & & & & \\
Caucasian & $59.0 \%$ & $50.0 \%$ & $47.1 \%$ & 0.556 \\
African American & $15.4 \%$ & $14.0 \%$ & $17.6 \%$ & 0.902 \\
Hispanic & $12.8 \%$ & $20.0 \%$ & $17.6 \%$ & 0.667 \\
Asian/Pacific Islander & $5.1 \%$ & $4.0 \%$ & $14.7 \%$ & 0.148 \\
Other & $7.7 \%$ & $12.0 \%$ & $2.9 \%$ & 0.327 \\
Past Medical History & & & & \\
Coronary Artery Disease & $25.6 \%$ & $38.0 \%$ & $35.3 \%$ & 0.452 \\
Diabetes Mellitus & $35.9 \%$ & $38.0 \%$ & $38.2 \%$ & 0.973 \\
Hypertension & $56.7 \%$ & $68.2 \%$ & $58.6 \%$ & 0.509 \\
\hline
\end{tabular}

Values represented as a mean \pm standard deviation or percentage (\%). 
Table 2 Pre-transplant factors.

\begin{tabular}{|c|c|c|c|c|}
\hline Transplant Factors & $\begin{array}{l}\text { Group A } \\
\text { PVR } \leq 1.6 \\
(n=39)\end{array}$ & $\begin{array}{l}\text { Group B } \\
1.6<\text { PVR } \leq 3 \\
(n=50)\end{array}$ & $\begin{array}{l}\text { Group C } \\
\text { PVR > } 3 \\
(n=34)\end{array}$ & $p$ value \\
\hline UNOS Status 1 at Transplant & $100.0 \%$ & $100.0 \%$ & $100.0 \%$ & 1.000 \\
\hline Cytomegalovirus Mismatch & $15.4 \%$ & $21.3 \%$ & $18.2 \%$ & 0.781 \\
\hline Creatinine $(\mathrm{mg} / \mathrm{dL})$ & $1.4 \pm 0.9$ & $1.4 \pm 0.8$ & $1.5 \pm 1.0$ & 0.884 \\
\hline Pre-transplant PRA $\geq 10 \%$ & $35.9 \%$ & $36.0 \%$ & $45.5 \%$ & 0.707 \\
\hline MPAP pre-MCS (mmHg) & $28.3 \pm 11.2$ & $36.1 \pm 11.0$ & $41.8 \pm 9.4$ & $<0.001$ \\
\hline \multicolumn{5}{|l|}{ MCS Device Type } \\
\hline LVAD & $53.8 \%$ & $52.0 \%$ & $72.7 \%$ & 0.091 \\
\hline BiVAD & $15.4 \%$ & $20.0 \%$ & $8.8 \%$ & 0.380 \\
\hline TAH & $30.8 \%$ & $28.0 \%$ & $17.6 \%$ & 0.407 \\
\hline $\begin{array}{l}\text { Time on Waiting List for Heart } \\
\text { Transplant (months) }\end{array}$ & $5.3 \pm 5.6$ & $7.3 \pm 7.3$ & $4.0 \pm 5.8$ & 0.068 \\
\hline $\begin{array}{l}\text { Time from MCS Device to Heart } \\
\text { Transplant (months) }\end{array}$ & $42.2 \pm 225.9$ & $37.1 \pm 199.3$ & $11.9 \pm 15.8$ & 0.884 \\
\hline
\end{tabular}


Table 3 Outcomes for heart transplant recipients with pulmonary hypertension bridged with mechanical circulatory support.

\begin{tabular}{|c|c|c|c|c|}
\hline Endpoints & $\begin{array}{l}\text { Group A } \\
\text { PVR } \leq 1.6 \\
(n=39)\end{array}$ & $\begin{array}{l}\text { Group B } \\
1.6<\text { PVR } \leq 3 \\
(n=50)\end{array}$ & $\begin{array}{l}\text { Group C } \\
\text { PVR > } 3 \\
(n=34)\end{array}$ & $p$ value \\
\hline 1-Year Survival & $97.4 \%$ & $90.0 \%$ & $91.2 \%$ & 0.392 \\
\hline $\begin{array}{l}\text { 1-Year Freedom from Moderate- } \\
\text { Severe TR }\end{array}$ & $94.9 \%$ & $82.0 \%$ & $70.6 \%$ & 0.016 \\
\hline 1-Year Freedom from CAV & $94.9 \%$ & $96.0 \%$ & $94.1 \%$ & 0.972 \\
\hline 1-Year Freedom from NF-MACE & $94.9 \%$ & $98.0 \%$ & $100.0 \%$ & 0.365 \\
\hline $\begin{array}{l}\text { 1-Year Freedom from Any-Treated } \\
\text { Rejection }\end{array}$ & $92.3 \%$ & $84.0 \%$ & $100.0 \%$ & 0.016 \\
\hline $\begin{array}{l}\text { 1-Year Freedom from Acute Cellular } \\
\text { Rejection }\end{array}$ & $97.1 \%$ & $97.7 \%$ & $100.0 \%$ & 0.625 \\
\hline $\begin{array}{l}\text { 1-Year Freedom from Antibody- } \\
\text { Mediated Rejection }\end{array}$ & $100.0 \%$ & $98.0 \%$ & $100.0 \%$ & 0.470 \\
\hline $\begin{array}{l}\text { 1-Week Post-Transplant PVR (wood } \\
\text { units) }\end{array}$ & $1.3 \pm 0.8$ & $1.7 \pm 1.0$ & $1.7 \pm 1.0$ & 0.162 \\
\hline 1-Year Creatinine (mg/dl) & $1.6 \pm 1.7$ & $1.6 \pm 0.9$ & $1.3 \pm 0.5$ & 0.163 \\
\hline 1-Year Total Bilirubin (mg/dL) & $0.6 \pm 0.4$ & $0.7 \pm 0.4$ & $0.5 \pm 0.3$ & 0.183 \\
\hline
\end{tabular}

TR: Tricuspid Regurgitation; CAV: Cardiac Allograft Vasculopathy; NF-MACE: Non-Fatal Major Adverse Cardiac Event;

Survival and freedom from event analysis were performed using the Kaplan-Meier method and compared between groups using log-rank tests.

Values represented as a mean \pm standard deviation or percentage (\%) 


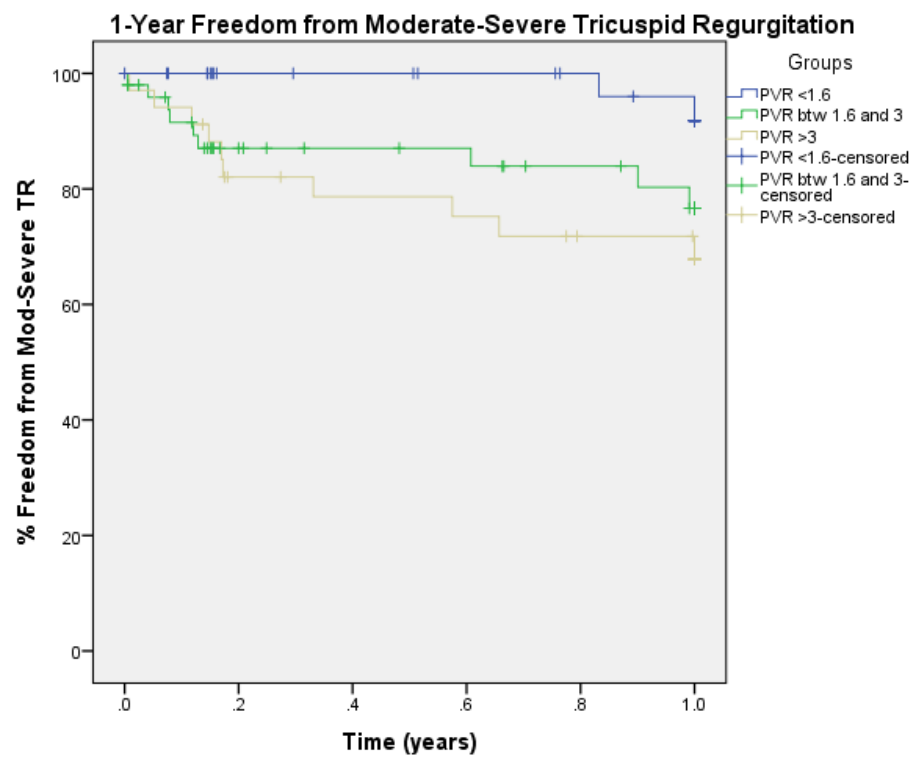

Figure 1 One-year freedom from moderate-severe tricuspid regurgitation Kaplan-Meier analysis. 1-year freedom from moderate-severe tricuspid regurgitation for hearttransplant recipients with pulmonary hypertension (PVR $\leq 1.6$ wood units, blue; $1.6<P V R \leq 3$, green; PVR>3, gold) that were placed on mechanical circulatory devices prior to transplant. Hatch marks denote censoring.

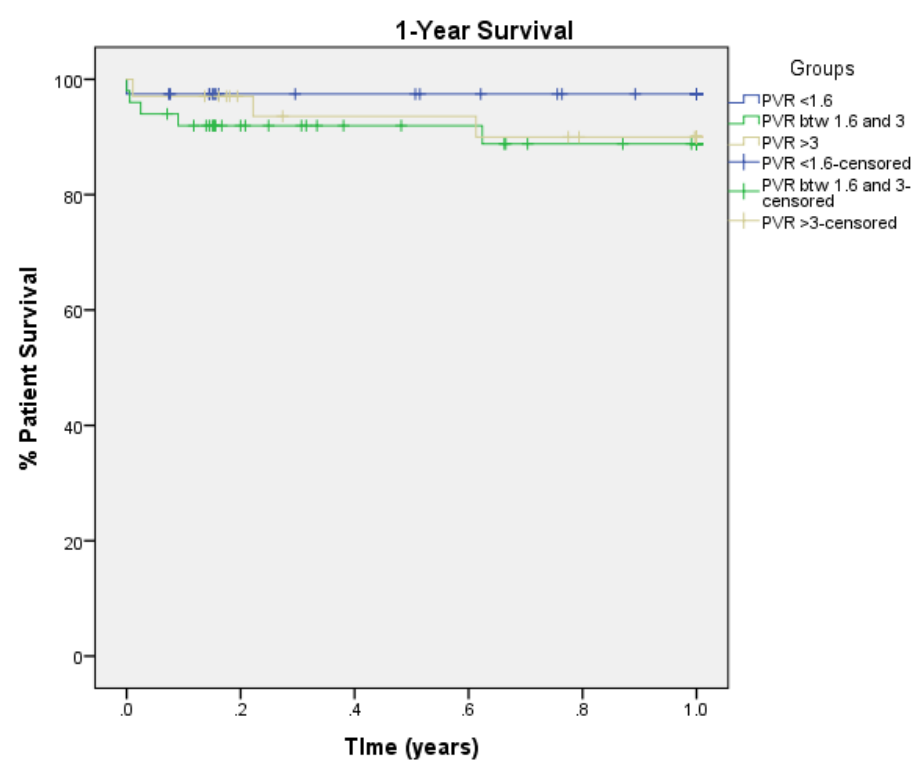

Figure 2 One-year survival Kaplan-Meier analysis. 1-year survival outcome for hearttransplant recipients with pulmonary hypertension (PVR $\leq 1.6$ wood units, blue; $1.6<P V R \leq 3$, green; PVR $>3$, gold) that were placed on mechanical circulatory devices prior to transplant. Hatch marks denote censoring. 


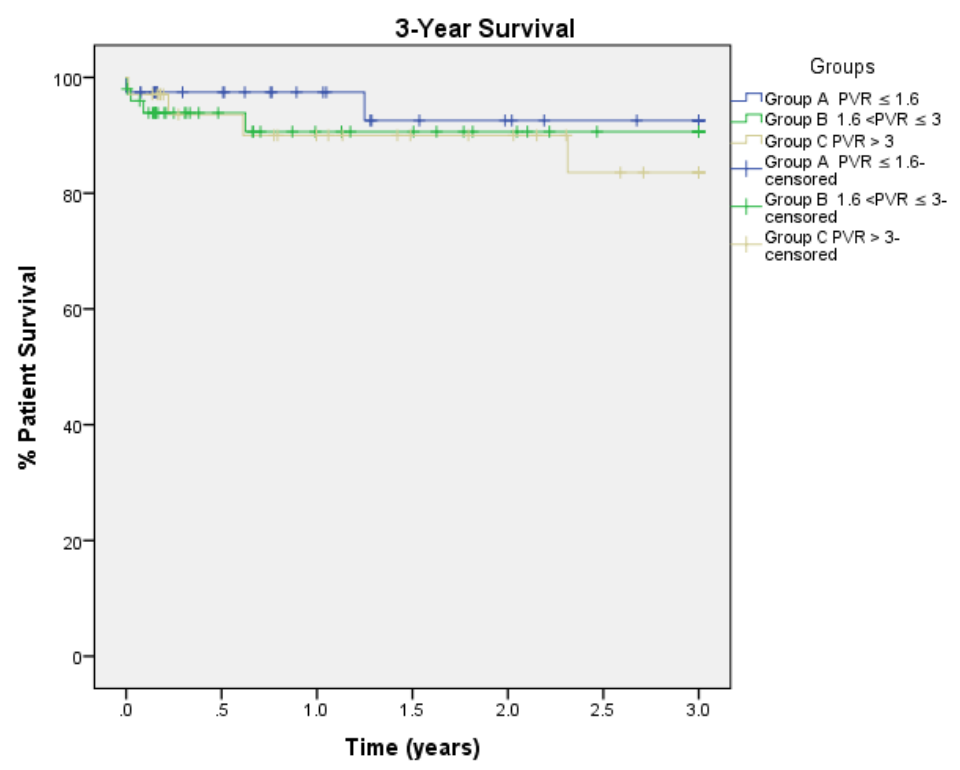

Figure 3 Three-year survival Kaplan-Meier analysis. 3-year survival outcome for hearttransplant recipients with pulmonary hypertension (PVR $\leq 1.6$ wood units, blue; $1.6<P V R \leq 3$, green; PVR $>3$, gold) that were placed on mechanical circulatory devices prior to transplant. Hatch marks denote censoring.

Table 4 1-Year survival stratified by no/mild tricuspid regurgitation vs moderate-severe tricuspid regurgitation.

\begin{tabular}{llll}
\hline & $\begin{array}{l}\text { No/Mild TR } \\
(\mathbf{n = 1 0 2 )}\end{array}$ & $\begin{array}{l}\text { Mod-Severe TR } \\
(\mathbf{n = 2 1 )}\end{array}$ & $\boldsymbol{p}$ value \\
\hline 1-Year Survival & $96.1 \%$ & $76.2 \%$ & $<0.001$ \\
\hline
\end{tabular}

TR: Tricuspid Regurgitation

Survival was performed using the Kaplan-Meier method and compared between groups using log-rank tests.

Table 5 1-year survival for patients with moderate-severe tricuspid regurgitation.

\begin{tabular}{lcccc}
\hline & $\begin{array}{c}\text { Group A } \\
\text { PVR } \leq \mathbf{1 . 6} \\
(\mathrm{n}=\mathbf{2})\end{array}$ & $\begin{array}{c}\text { Group B } \\
\mathbf{1 . 6}<\text { PVR } \leq \mathbf{3} \\
(\mathrm{n}=\mathbf{9})\end{array}$ & $\begin{array}{c}\text { Group C } \\
\text { PVR }>\mathbf{3} \\
(\mathrm{n}=\mathbf{1 0 )}\end{array}$ & $\boldsymbol{p}$ value \\
\hline $\begin{array}{l}\text { 1-Year Survival for Patients with } \\
\text { Moderate-Severe TR }\end{array}$ & $100.0 \%$ & $77.8 \%$ & $70.0 \%$ & 0.788 \\
\hline
\end{tabular}

TR: Tricuspid Regurgitation

Survival was performed using the Kaplan-Meier method and compared between groups using log-rank tests. 


\section{Discussion}

Patients with advanced heart failure and $\mathrm{PH}$ continue to represent a challenging population, with limited options for advanced therapies. These patients historically experience very poor outcomes when taken directly to $\mathrm{OHT}$, leading to the understanding that $\mathrm{PH}$ is a relative contraindication for $\mathrm{OHT}[1,2]$. Recently, implantation of MCS as a bridge to OHT in patients with PH has been established as a viable alternative, with a resultant decrease in PVR and restoration of candidacy in select patients [3-5].

It has been generally understood that the prolonged elevated left atrial pressures and subsequent increase in resistance within the pulmonary circulation is the driving factor behind $\mathrm{PH}$ in this population, a manifestation of remodeling within the pulmonary vascular system [6, 7]. It is this remodeling that ultimately leads to the poor outcomes following OHT in this population, as explantation of the native heart and subsequent reduction in left atrial pressures does not result in meaningful change to the resistance seen by the implanted donor right heart. The unconditioned donor heart is unable to tolerate this resistance, and the subsequent right heart failure that results is typically catastrophic.

The threshold at which a transplant center may consider $\mathrm{PH}$ a contraindication for OHT varies depending on the degree of risk an individual center may tolerate, but generally stands at around 3 WU in most US centers $[1,8]$. Patients with prohibitive $\mathrm{PH}$ and heart failure who are not candidates for OHT have historically had poor long-term outcomes. The potential to bridge to transplantation and restore candidacy in these individuals after placement of a MCS device is an encouraging prospect, and the data included here, as well as previously reported results [4], indicate that outcomes among these patients are good. Though good outcomes following MCS for OHT patients with $\mathrm{PH}$ have been previously reported, the prior data focuses on patients with LVADs specifically. To our knowledge this is the first study to include BiVAD and TAH patients, and analysis of survival out to 3 years continues to be positive (Figure $3, p=0.681$ ). These generally favorable results, including good one-year and three-year survival, indicate that where univentricular support (LVAD) is insufficient, $\mathrm{PH}$ patients undergoing biventricular MCS (BiVAD or TAH) as a bridge to OHT should be viewed as potentially viable candidates even with high baseline PVR. It is notable that the observed rates of biventricular support were lowest in the group with the highest PVR. The reason for this observation may be that patients who have the highest PVR simply do not survive to MCS implantation if their RV function is inadequate, and thus this group is selected for patients that are less likely to need RV support. Additionally, we suspect that given the significantly larger surgical burden of biventricular support vs. univentricular support, the surgical team may have been biased against placing biventricular support in patients with higher baseline PVR.

Though the general trend in this study was towards good outcomes among all groups $(92.7 \%$ cumulative one-year survival), there were differences noted among the rates of moderate-severe TR, with a trend towards worse rates corresponding with worsening pre-VAD PVR. This increased rate of moderate-severe TR was seen despite early improvement in PVR at the 1-week cardiac catheterization for hemodynamic data among all groups post-transplantation (1.3, 1.7, and 1.7 for groups A, B, and C, respectively). Unfortunately, our data set did not include PAH therapies employed 
in the post-operative period and at the time of this catheterization (such as inhaled nitric oxide), longterm PAH therapies, nor did we have the data to longitudinally follow the PVR over time by hemodynamic catheterization or echo. While it appears that the PVR normalized post-OHT, we were intrigued to see that the patients with higher pre-operative PVR had higher degrees of moderatesevere TR. It is unclear, from a pathophysiology standpoint, what the underlying mechanism for increased rates of moderate-severe TR is, given this observed early improvement in PVR but it is likely associated with abnormal underlying pulmonary vascular pathology. Moreover, associated RV dysfunction in this population was not specifically examined, and as such the association of TR and RV dysfunction is also unclear. Given these limitations, we cautiously analyze this data and the conclusions we draw from it.

The long-term consequence of moderate-severe TR does not seem to be benign, as the survival difference between those with and without moderate-severe TR is significant $(76.2 \%$ vs $96.1 \%$ at 1 year, $p<0.001$ ). This finding is similar to those found previously indicating a deleterious effect of moderate-severe TR on survival after OHT [9]. It should be noted that the aforementioned study by Wartig, et al., as well as another work by Herrmann, et al. [10], did not identify pre-operative PVR (immediately prior to OHT) as a risk factor for development of TR following OHT. In this study we do note a statistically-significant increase in the rate of moderate-severe TR following OHT as the preMCS implantation PVR rises, something that was not specifically evaluated in the prior works. This finding indicates a relationship between pre-MCS PVR and TR, and in doing so at least suggests an association between pre-MCS PVR and risk of death for these patients, though this is not statistically significant in our data, possibly due to a lack of power. It should be noted that, to the best of our knowledge, none of the TR seen was related to iatrogenic injury (during biopsy, line placement, etc.).

Though rates of moderate-severe TR were greatest in patients with the highest baseline PVR, the rate of any-treated rejection was lowest, and was statistically significant in difference between groups (Table 3). It is unclear at this time what causal relationship could be influencing this finding, especially as there did not seem to be a linear relationship between baseline PVR and rejection.

\section{Limitations}

The above study is limited by its retrospective design, the short-term follow up, and by the small cohort (123 total patients). These limitations cause an increased risk for type II error. Further study in the future with larger cohorts and greater follow up is needed. Additionally, there are factors that were not captured in this dataset that may have influenced the results. For example, the use of inhalational pulmonary vasodilators or oral phosphodiesterase inhibitors post-operatively was not captured, though both did occur.

\section{Conclusions}

In conclusion, MCS implantation (including biventricular support systems) as a bridge to $\mathrm{OHT}$ has good one-year outcomes among patients with increasing pre-MCS PVR. However, patients with very elevated PVR prior to MCS implantation have an increased rate of moderate-severe TR posttransplant. Among all MCS to OHT patients, moderate-severe TR after OHT has an associated 
increased risk of early death. The relationship between these findings requires further study with larger cohorts and longer follow up periods.

\section{Acknowledgments}

We would like to acknowledge the Cedars-Sinai Smidt Heart Institute for continued support.

\section{Author Contributions}

$\mathrm{DE}$, and FE devised the project, oversaw and assisted with data analysis, and wrote the manuscript; $\mathrm{RL}$ was responsible for data analysis and data acquisition; JK, JM, DM, JC, DR, and AT aided in results interpretation and aided in manuscript creation.

\section{Funding}

No external funding sources were provided for this study. All authors attest that there are no relevant financial relationships with an external or commercial entity to disclose.

\section{Competing Interests}

The authors have declared that no competing interests exist.

\section{References}

1. Mehra MR, Kobashigawa J, Starling R, Russell S, Uber PA, Parameshwar J, et al. Listing criteria for heart transplantation: International society for heart and lung transplantation guidelines for the care of cardiac transplant candidates-2006. J Heart Lung Transpl. 2006; 25: 1024-1042.

2. Delgado JF, Gómez-Sánchez MA, Sáenz de la Calzada C, Sánchez V, Escribano P, HernándezAfonso J, et al. Impact of mild pulmonary hypertension on mortality and pulmonary artery pressure profile after heart transplantation. J Heart Lung Transpl. 2001; 20: 942-948.

3. Mikus E, Stepanenko A, Krabatsch T, Loforte A, Dandel M, Lehmkuhl HB, et al. Reversibility of fixed pulmonary hypertension in left ventricular assist device support recipients. Eur J Cardiothorac Surg. 2011; 40: 971-977.

4. Moayedifar R, Zuckermann A, Aliabadi-Zuckermann A, Riebandt J, Angleitner P, Dimitrov K, et al. Long-term heart transplant outcomes after lowering fixed pulmonary hypertension using left ventricular assist devices. Eur J Cardiothorac Surg. 2018; 54: 1116-1121.

5. Kutty RS, Parameshwar J, Lewis C, Catarino PA, Sudarshan CD, Jenkins DP, et al. Use of centrifugal left ventricular assist device as a bridge to candidacy in severe heart failure with secondary pulmonary hypertension. Eur J Cardiothorac Surg. 2013; 43: 1237-1242.

6. Guazzi M, Arena R. Pulmonary hypertension with left-sided heart disease. Nat Rev Cardiol. 2010; 7: 648-659. 
7. Zimpfer D, Zrunek P, Roethy W, Czerny M, Schima H, Huber L, et al. Left ventricular assist devices decrease fixed pulmonary hypertension in cardiac transplant candidates. J Thorac Cardiovasc Surg. 2007; 133: 689-695.

8. Wartig M, Tesan S, Gäbel J, Jeppsson A, Selimovic N, Holmberg E, et al. Tricuspid regurgitation influences outcome after heart transplantation. J Heart Lung Transpl. 2014; 33: 829-835.

9. Herrmann G, Simon R, Haverich A, Cremer J, Dammenhayn L, Schäfers HJ, et al. Left ventricular function, tricuspid incompetence, and incidence of coronary artery disease late after orthotopic heart transplantation. Eur J Cardiothorac Surg. 1989; 3: 111-117.

10. Delgado JF, Conde E, Sánchez V, López-Ríos F, Gómez-Sánchez, Escribano P, et al. Pulmonary vascular remodeling in pulmonary hypertension due to chronic heart failure. Eur $\mathrm{J}$ Heart Fail. 2005; 7: 1011-1016.

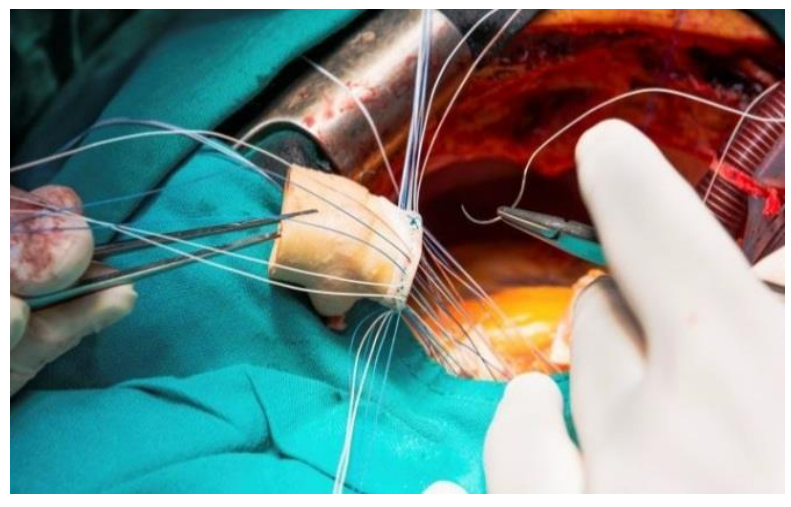

Enjoy OBM Transplantation by:

1. Submitting a manuscript

2. Joining in volunteer reviewer bank

3. Joining Editorial Board

4. Guest editing a special issue

For more details, please visit:

http://www.lidsen.com/journals/transplantation 